\title{
Remote Sensing Image Fusion for Unsupervised Land Cover Classification
}

\author{
Chaabane Ferdaous \\ University of $7^{\text {th }}$ November at Carthage, Higher school of Communications of Tunis \\ Sup'Com, URISA \\ Tunisia
}

\section{Introduction}

\subsection{Context}

With the development of new satellite systems and the accessibility of data from public through web services like Google Earth, remote sensing imagery, knows today an important growing which advanced and still advances researches in this area on different aspects. Especially in cartography, many studies have been conducted for multi-source satellite images classification. These studies aim to develop automatic tools in order to facilitate the interpretation and provide a semantic land cover classification.

Classical tools based on satellite images deal essentially with one category of satellite images which allows a partial interpretation. Multi-sensor or multi-source image fusion have been applied in the field of remote sensing since 20 years and continues today to provide efficient solutions to problems related to detection and classification. The work presented in this chapter is a part of multi-source fusion research efforts to have reliable and automatic satellite image interpretation. We propose to apply the new fusion concepts and theories for multi-source satellite images. Our main motivation is to measure the real contribution of multi-source image fusion according to the exploitation of satellite images separately.

Recent studies suggest that the combination of imagery from satellites with different spectral, spatial, and temporal information may improve land cover classification performance. The use of multi-source satellites images fully take into account the complementary and supplementary information provided by different data sources and considerably optimize the classification of cartographic objects. Particularly, combination of optical and radar remote sensing data may improve the classification results because of the complementarities of these two sources. Spectral features extracted form optical data may remove some difficulties faced when using only radar images. However, radar images present the following massive advantage: the possibility of penetrating the clouds. Thus, data fusion technique is applied to combine these two kinds of information.

\subsection{Proposed approach}

In literature, there is a huge variety of fusion theories mainly probabilistic and Bayesian theory [Mitchell, 2007], fuzzy and possibility theory [Milisavljevi'C \& Bloch, 2009], Dumpster and Shafer theory, etc. [Milisavljević \& Bloch, 2008]. However, most of them are investigated in four steps which are: modeling, estimation, combination and decision (cf. 
Fig. 1.). For radar and optical images fusion, we choose to apply a Bayesian fusion framework in order to take into account the speckle radar texture which can be better represented by a Markovian gamma distribution [Rui-hui et al., 2009].

The originality of the proposed method is on one hand, the introduction of spatial and contextual information in fusion process using Markovian modeling with an optimal neighborhood order. Indeed, it has been shown [Meddeb et al., 2007] that the optimal neighborhood order allows a better representation of the speckle radar texture in terms of contrast, homogeneity, isotropy, etc. On the other hand, the given approach characterizes the radar texture data with a Markovian gamma auto-model. The radar texture is being usually modeled by a Gaussian model in probabilistic fusion processes.

Fig. 1. presents the main steps for multi-source image classification. As we can see, before applying fusion processing, some pretreatments must be applied to both satellites data due to the different nature of optical and radar images. The first pretreatment is the geometric correction which allows the superposition of the two remote sensing images [Zitova \& Flusser, 2003]. The second pretreatment is the single image classification applied to both radar and optic images using a Fuzzy C-Means (FCM) algorithm [Wang, 1990]. Radar images are gamma MAP [Hosomura \& Jayasekera, 1993] filtered before classification in

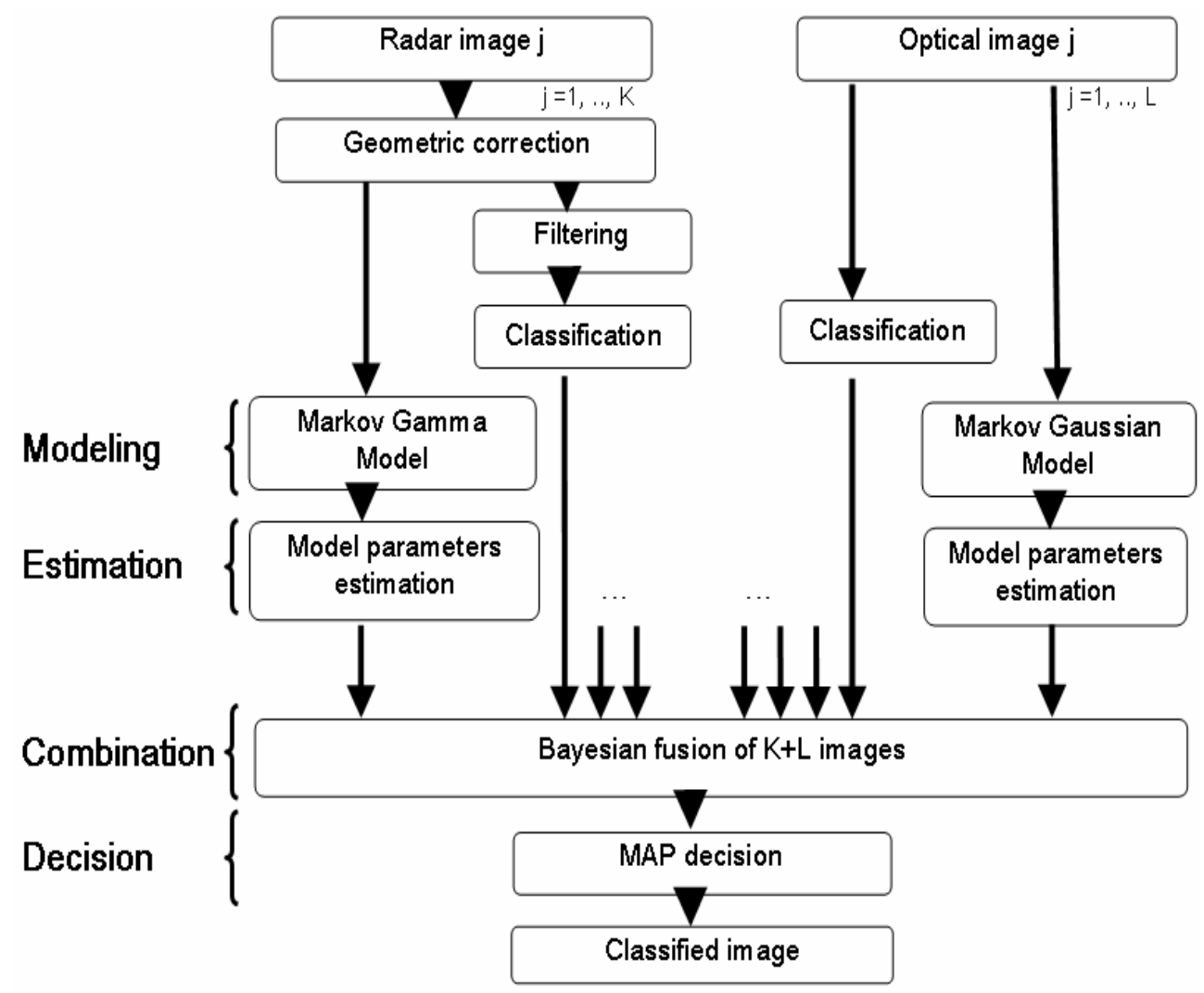

Fig. 1. Multi-source probabilistic fusion approach for land cover classification: main steps. 
order to smooth the granular texture and reduce speckle noise. For each pixel, we model its posterior probability by a Besag Markov Gaussian auto-model in optical case and a Besag Markov gamma auto-model in radar case [Besag, 1974]. Parameters models are estimated using Expectation Maximization (EM) algorithm [Hogg et al., 2005]. Then the posterior probabilities are combined by the way of Bayesian fusion theory [Bloch, 2008].

This chapter is organized as follows. First, we describe briefly the three pretreatments phases. Secondly, the posterior probability modeling is presented for both radar and optical images. These probabilities are then used to present the Bayesian fusion process. Finally, pretreatments and fusion results are exposed in order to show land cover classification performances. Qualitative and Quantitative evaluations of the obtained results are also presented.

\section{Fusion preprocessing}

The exploitation of multi-source satellite images allows the obtaining of new signatures. However, these images are generated from various sensors, have different features (geometric, resolution, lighting, etc.) and are mainly not associated to the pixel level. Fusion process appears then complex and very sensitive to these data. To deal with this problem, some pretreatments must be done before combination step in order to correct images and prepare them for a simultaneous exploitation.

These preprocessing steps are essentially:

- Geometric correction

- Filtering

- Single image classification

- Data representation or modeling.

\subsection{Geometric correction}

The first pretreatment is the geometric superposition and geocoding [Hong \& Schowengerdt, 2005]. For both optical and radar images, acquisition process is not the same and the measured data have different natures. Because of sampling and oblique geometric acquisition in radar imagery, there is no direct transformation from radar to optical image and inversely. Several registration techniques exist. Each registration method is characterized by four criteria that are essentially:

- The attributes: these are features extracted from both images to guide the transformation. There are extrinsic attributes (e.g. fixed external markers) and intrinsic attributes (e.g. the grayscale or extracted geometric primitives).

- The similarity criterion: it sets a certain distance between images attributes to quantify the notion of similarity.

- The deformation model: it determines how the image is geometrically changed. It can be local or global, and is characterized by a certain number of degrees of freedom.

- The optimization strategy: it determines the best processing within the meaning of a certain similarity criteria and a deformation model.

Depending on the type of deformation model, there are two types of registration: rigid and elastic registration [Shabou et al., 2007]. Among rigid registration family, there are linear or nonlinear transformations. The control points based registration is a non linear approach for which the geometric correction is determined according to a polynomial model (deformation model). The polynomial coefficients are calculated by minimizing the 
geometric errors between two sets of control points selected manually in both images (optimization strategy). These points should be visible on the two images. The quality of the geometric correction depends on the precision of these points's localization, their distribution in the image and their number. More, are the marked points, better is the correction. The polynomial transformation is then performed projecting one image onto another.

\subsection{Radar texture filtering}

The exploitation of radar images in terms of land cover classification presents some difficulties mainly because of the speckle noise.

The Synthetic Aperture Radar (SAR) is a coherent imaging system where backscattered signals coming from multiple distributed targets may interfere in any point of the space. If the interference is constructive, it results a brilliant point otherwise a dark point. The speckle noise, which gives the SAR image a granular character, reduces the correlation between pixels increasing thus the variance and the mean radar reflectivity of a local area. This phenomenon is a serious problem that degrades the quality of SAR images and causes difficulties for targets detection thus image interpretation. It is often compared to a multiplicative noise i.e. in direct proportion of the radar reflectivity which increases the difficulty of completely eliminating it.

It appears therefore necessary to reduce the speckle noise before using SAR images. Many techniques exist in the literature. Two techniques are often used: the multi-look processing, usually done at acquisition time, averages out the speckle noise by taking several "looks" at a single pixel of the radar image and the spatial filtering technique which includes adaptive and non-adaptive filters, is applied locally on a neighborhood around each pixel. The optimal choice of a filter depends on the ability of this filter to reduce speckle noise when preserving radiometric and radar texture information. The non-adaptive filters apply the same weights uniformly across the entire image thus they do not take into account backscattered signal local properties (example, the median and simple mean filters).

The adaptive filters adapt their weights across the image to the speckle level. They explicitly take account of the speckle and integrate local backscattering properties in their mathematical models. There are many forms of adaptive speckle filtering [Lee et al., 1994], including the Lee filter, the Frost filter, and the Gamma Maximum-A-Posteriori (GMAP) filter [Baraldi \& Panniggiani, 1995]. The last one is based on the assumption that the radar intensity follows a gamma distribution. This filter, relatively to other filters, improves detection of edges and details in high-texture areas using second order spatial statistics and without losing information. Many other filters have been recently introduced [Maître, 2000] [Lee et al., 1994] but they have all comparable smoothing effects.

\subsection{Single image classification}

A critical step of multi-source satellite images processing is classification, whose objective is to identify all land cover types. There are mainly two categories of classification techniques:

- The supervised classification: it relies on prior information knowledge to search for classes. Training areas corresponding to sample pixels that are representative of specific classes, are selected manually by the user who also designates the outputs. The classification system is then used to develop a statistical characterization of each class basing on the training samples. The image is then classified by examining each pixel 
and making a decision about which of the signatures it is closest to. The most known supervised classification methods include neuronal network [Benediktsson et al., 1990] and SVM based approaches [Bazi \& Melgani, 2006].

- The non-supervised classification: it uses data discriminating features to separate pixels in different classes as homogeneous as possible. The number of classes is often unknown by the user. These automatic methods are usually iterative and construct gradually classes basing on distances or pseudo-distances. Among these methods, we can mention the K-means algorithm [Philips, 2002] which has been largely used. Then, the "ISODATA (Iterative Self Organizing Data Analysis) clustering" algorithm [Philips, 2002], the FCM (Fuzzy C-means) method [Wang, 1990] [Bezdek et al., 1984], the "Competitive Learning" technique [Tang, 1998], etc. In the presented work, we focused on two non-supervised classification methods which have been used for satellite images: "ISODATA clustering" and FCM algorithm. The ISODATA algorithm is similar to the k-means algorithm with the distinct difference that the number of clusters is not previously known. It minimizes the distance to the mean as method of clustering and iterates through the data until user specified thresholds are reached and the optimal set of output classes is obtained. The ISODATA algorithm is very sensitive to initial starting values. Another commonly used unsupervised classification method is the FCM algorithm which is very similar to K-Means, but fuzzy logic is incorporated and recognizes that class boundaries may be imprecise or gradational. The FCM classification method creates an initial set of prototype classes and then determines a membership grade for each class for every pixel. The grades are used to adjust the class assignments and calculate new class centres, and the process is repeated until the iteration limit is reached. The FCM algorithm is more adaptive than other hard clustering methods and performs extremely well in situations of large variability of cluster shapes, densities and number of data points in each cluster.

Each optical and filtered radar image is classified using an automatic unsupervised FCM algorithm [Wang, 1990] to allow cartographic objects detection and classification. The results of FCM algorithm classification constitute the input of the fusion process.

\subsection{Data representation}

The exploitation of spatial information is fundamental for image processing, more particularly in image fusion. We often require specific developments to adapt the methods for each application. In the context of this work, we aim to introduce spatial information at the level of combination in fusion processing. Probabilistic Markov Random Fields (MRF) offer a natural framework to this. Markovian modelling implies that the probability that a random variable, in a pixel takes a given value knowing the entire image is equal to the probability in this pixel knowing its neighbours. It allows thus describing spatial interaction between level's pixels, by their neighbor's graph which coverage is quantified by a field order.

Previous works [Decombes et al., 1999] [Lorette et al., 2000] show Markov models effectiveness for texture and region characterization. Besag Markovian auto-models [Besag, 1974] form a class of Markov Random fields particularly simple and useful for spatial statistics. They are based on conditional distributions which are assumed to belong to an exponential family.

A Besag auto-model is defined as a Markovian field associated to Gibbs energy by: 


$$
U(x)=\sum_{s \in S} \phi_{1}\left(x_{s}\right)+\sum_{(s, r) \in C_{2}} \phi_{2}\left(x_{s}, x_{r}\right)
$$

Where $x_{s}$ is the current pixel, $S$ is the whole set of pixels in the image, $C_{2}$ represents the set of all possible order 2 cliques, $x_{r}$ are order 2 neighborhood pixels of pixel $x_{s}$. Both $\Phi_{1}$ and $\Phi_{2}$ characterize totally the Markovian field:

$\phi_{1}\left(x_{s}\right)$ is the data description potential.

$\phi_{2}\left(x_{s}, x_{r}\right)$ is the interaction potential between $x_{s}$ and $x_{r}$.

Order 2 is the lowest order to convey contextual information. It is widely used because of its simple formulation and low computational cost. However, previous works [Meddeb et al., 2007] show that superior orders neighborhoods allow a better representation of the optical and the radar texture. The optimal neighborhood order is determined basing on descriptors such as contrast, homogeneity, isotropy, entropy, texture coefficients, etc. Experimental results (cf. Fig. 2.) showed a convergence of descriptors majority to order 4 . However, the obtained curves show a small loss of performances between order 3 and 4 . For this reason and due to calculation complexity, we choose the third order neighborhood.
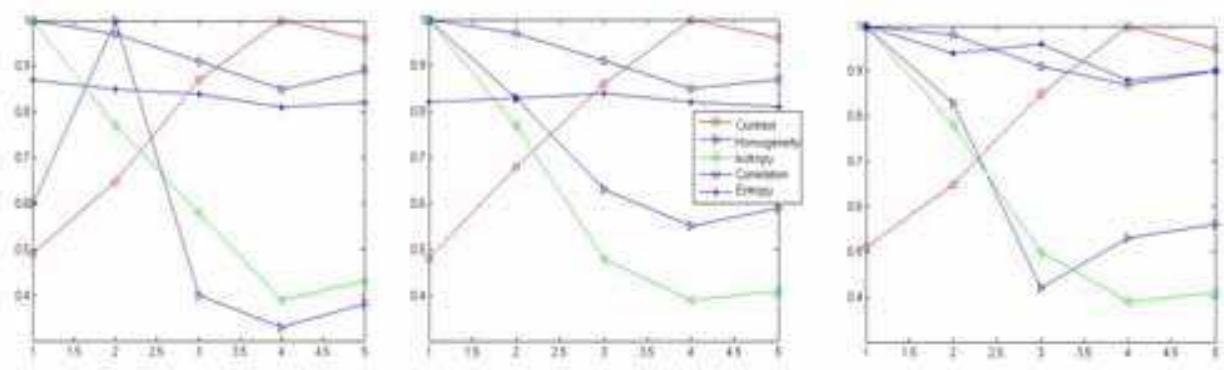

Fig. 2. Radar texture features versus neighborhood order for three region of interest: Left (water area), middle (Urban area), right (vegetation area) [Meddeb et al., 2007]

The auto-models can be classified according to the energy potential $\Phi_{1}$ i.e. to assumptions made about $x_{s}$ probability laws. Among these models, we can distinguish the auto-logistic, the auto-binomial, the auto-normal and the auto-gamma models. The following describes briefly the two last MRF models for representing respectively optical and radar image textures.

\subsubsection{Auto-normal model}

An auto-normal model also called Gaussian MRF is much used in the literature especially for segmentation, restoration and regularization problems. The corresponding energy is of the following form:

$$
U(x)=\alpha \sum_{s \in S}\left\|x_{s}-\mu_{s}\right\|^{2}+\beta \sum_{(s, r) \in C}\left\|x_{s}-x_{r}\right\|^{2}
$$

Where $\mathrm{C}$ is the set of cliques around the pixel $x_{s}, \mu_{s}$ is the local mean and:

- $\quad \alpha \sum_{s \in S}\left\|x_{s}-\mu_{s}\right\|^{2}$ is the potential describing the data, 
- $\quad \beta \sum_{(s, r) \in C}\left\|x_{s}-x_{r}\right\|^{2}$ is the regularization term describing interaction between pixels.

The conditional probability density function (pdf) of the site s, is given by:

$$
P\left(X_{s}=x_{s} / X_{r}=x_{r}, r \in V_{s}\right)=N\left(\mu_{s}, \sigma_{s}^{2}\right)
$$

Where $X_{s}, X_{r}$ represent respectively the random variables associated to sites $s$ with value $x_{s}$ and $r$ of value $x_{r}$ and $V_{s}$ is the neighbourhood of the site $s$.

The mean and the variance of the site $s$ are defined by:

$$
\left\{\begin{array}{l}
\mu_{s}=E\left\{x_{s} / x_{r}, r \in V_{s}\right\}=m_{s}+\sum_{r \in V_{s}} \beta_{s r}\left(x_{r}-m_{r}\right) \\
\sigma_{s}^{2}=\operatorname{Var}\left\{x_{s} / x_{r}, r \in V_{s}\right\}
\end{array}\right.
$$

Where $m_{s}$ and $m_{r}$ are respectively the means around the site $s$ and $r$ and $\beta_{s r}$ is the interaction parameter between sites $s$ and $r$.

Thus the conditional probability becomes:

$$
P\left(X_{s}=x_{s} / X_{r}=x_{r}, r \in V_{s}\right)=\frac{\exp \left(-\frac{1}{2 \sigma^{2}}\left(x_{s}-m_{s}-\sum_{r \in V_{s}} \beta_{s r}\left(x_{r}-m_{r}\right)\right)^{2}\right)}{\sum_{s \in S} \exp \left(-\frac{1}{2 \sigma^{2}}\left(x_{s}-m_{s}-\sum_{r \in V_{s}} \beta_{s r}\left(x_{r}-m_{r}\right)\right)^{2}\right)}
$$

Where $\mu_{s}, \sigma_{s}$ and $\beta_{s r}$ are the normal auto-model parameters to be estimated.

Several works [Descombes et al., 1999] demonstrate that Gaussian MRF shows better representation of optical images mainly because of texture homogeneity of the most cartographic objects. Other works [Belhadj et al. 2000] showed that the auto-gamma model is more adapted to radar images than auto-normal one because of the granular nature of radar texture.

\subsubsection{Auto-gamma model}

The auto-gamma model takes into account simultaneously the radar and speckle texture which guarantees to this model a considerable advantage [Belhadj et al., 2000]. Indeed, it makes it possible to be free from the pretreatment step which is speckle filtering. However, filtering is necessary before single radar image classification to limit the number of classes and to regularize their contours.

The auto-gamma model law is given by:

$$
P\left(X_{s}=x_{s} / X_{r}=x_{r}, r \in V_{s}\right)=\gamma\left(a,\left(\alpha_{s}+\sum_{r \in V_{s}} \beta_{s r} x_{r}\right)\right)
$$

Where $a$ and $\alpha_{s}$ are the auto-gamma model parameters.

The local conditional probability becomes starting from this expression by:

$$
P\left(X_{s}=x_{s} / X_{r}=x_{r}, r \in V_{s}\right)=\frac{x_{s}^{a-1} \exp \left(-x_{s}\left(\alpha_{s}+\sum_{r \in V_{s}} \beta_{s r} x_{r}\right)\right)}{\sum_{s \in S} x_{s}^{a-1} \exp \left(-x_{s}\left(\alpha_{s}+\sum_{r \in V_{s}} \beta_{s r} x_{r}\right)\right)}
$$


Where $a, \alpha_{s}$ and $\beta_{s r}$ are the gamma auto-model parameters to be estimated.

\subsubsection{Parameter estimation}

One of the main tasks of Bayesian classification is parameters estimation. In order to estimate the auto-models parameters by the maximum likelihood method we use the Expectation-Maximizing (EM) algorithm. Proposed by Dumpster et al. [Dumpster et al., 1977], the EM algorithm is an iterative algorithm for the calculation of the estimator of the maximum likelihood parameter of a model. The EM algorithm proceeds in two steps: an expectation step, followed by a maximization step which are iterated until convergence.

Parameters estimation algorithm was applied on both auto-normal and auto-gamma simulated images in order to validate the estimation process.

\section{Probabilistic fusion model}

In this section, we present a definition of data fusion in the field of image processing as well as the principal fusion steps applied to multi-source images. We will especially focus on the Bayesian probabilistic approach which has been adopted in this work.

\subsection{Fusion steps}

In the literature, there are several definitions for data fusion. Most of them are quoted in [Bloch, 2008] [Klein, 2004]. The definition that we adopt here was introduced by Bloch in [Bloch, 2008] and is adapted to the case of multi-source images: "The information fusion consists in combining heterogeneous information resulting from several sources in order to improve the decision." This definition is sufficiently general to include the diversity of fusion problems in signal and image processing.

Fusion is not usually a simple task. It can be investigated into four steps. We describe them briefly here, because they will be used for the presentation of fusion Bayesian theory. Let us consider a general fusion problem for which one has $K$ sources, $S_{1}, S_{2}, \ldots, S_{K}$ and for which the goal is to make a decision chosen from $N$ possible decisions $d_{1}, d_{2}, \ldots, d_{N}$. The principal steps necessary to build fusion process are as follows [Bloch, 2008]:

- Modelling

- Estimation

- Combination

- Decision.

1. Modelling: this step includes the formalism choice and the mathematical expressions to be connected to this formalism. This step can be guided by additional or prior information about the context or the field of study. Let us suppose that each source $S_{j}$ provides information represented by the model $M_{i j}$ for the decision $d_{i}$. The shape of $M_{i j}$ depends of course on the selected formalism.

2. Estimation: the majority of modelling techniques require a parameters estimation phase (for example all the distributions based methods). Here also additional information can be used.

3. Combination: this step relates on the choice of a compatible operator to the modelling formalism. It is also guided by additional information.

4. Decision: it represents the crucial fusion step, which makes it possible to change the information (provided by the sources) to the choice of a decision $d_{i}$. 
The way in which these stages are arranged defines the fusion system and its architecture. In the literature, there are several fusion approaches. We focus here on probabilistic fusion theory and describe in details its main steps.

\subsection{Bayesian fusion theory}

The probabilistic fusion theory is the most useful fusion tool which is associated to Bayesian decision theory. This approach treats information uncertainty and is based on solid mathematical tools.

\section{- Modelling}

Information in probabilistic theory is modelled by a conditional probability. For example, the probability that a pixel $x$ belongs to a particular class $C_{i}$, given the available image $I_{j}$ has the following form [Bloch, 2008]:

$$
M_{i}^{j}(x)=p\left(x \in C_{i} / I_{j}\right)
$$

This probability is calculated starting from the information extracted from the image features $f_{j}(x)$. In the simplest case, it can be the considered pixel grey level, or more complex information requiring some pretreatments. The previous equation does not then depend any more on the entire image $I_{j}$ and is written in the simplified form as:

$$
M_{i}^{j}(x)=p\left(x \in C_{i} / f_{j}(x)\right)
$$

\section{- Estimation}

In absence of strong functional modelling of the observed phenomena, probabilities $M_{i}^{j}(x)=p\left(f_{j}(x) / x \in C_{i}\right)$ or more generally $M_{i}^{j}(x)=p\left(I_{j} / x \in C_{i}\right)$ represents the conditional probability according to class $C_{i}$, of the information provided by the image $I_{j}$. They are learned or estimated by enumeration on test areas (the simplest case) or by training on these areas the parameters of a given probabilistic law.

\section{- Combination within a Bayesian framework}

Once information resulting from each sensor, represented by a convenient model, they can be combined according to specific rules according to the selected theoretical framework. The probabilistic and Bayesian fusion can be carried out by two equivalent ways and at two different levels [Bloch, 2008]:

- The fusion can be done at the modelling step. Then we calculate probabilities for $l$ images sources as $p\left(x \in C_{i} / I_{1}, \ldots, I_{l}\right)$. Using the Bayes rule:

$$
p\left(x \in C_{i} / I_{1}, \ldots, I_{l}\right)=\frac{p\left(I_{1}, \ldots, I_{l} / x \in C_{i}\right) p\left(x \in C_{i}\right)}{p\left(I_{1}, \ldots, I_{l}\right)}
$$

The different terms are estimated by training.

- The fusion can also be done using Bayes rule itself. The information resulting from a source comes to update the information estimated according to the preceding sources:

$$
p\left(x \in C_{i} / I_{1}, \ldots, I_{l}\right)=\frac{p\left(I_{1} / x \in C_{i}\right) \ldots p\left(I_{l} / x \in C_{i}, I_{1}, \ldots I_{l-1}\right) p\left(x \in C_{i}\right)}{p\left(I_{1}\right) p\left(I_{1} / I_{2}\right) \ldots p\left(I_{l} / I_{1}, \ldots, I_{l-1}\right)}
$$


Very often, known the complexity of the training starting from several sensors and the difficulty of obtaining sufficient statistics, these equations are simplified under the independence assumption. Several criteria were proposed to check the validity of this assumption. The previous formula becomes then:

$$
p\left(x \in C_{i} / I_{1}, \ldots, I_{l}\right)=\frac{\prod_{j=1}^{l} p\left(I_{j} / x \in C_{i}\right) p\left(x \in C_{i}\right)}{p\left(I_{1}, \ldots, I_{l}\right)}
$$

The equation (12) revealed clearly the type of information combination as a product. We can notice also that the prior probability $p\left(x \in C_{i}\right)$ plays the same role as the sources in the combination. Let us mention here that the Bayesian combination has a conjunctive character [Bloch, 2008] by the means of multiplication.

\section{- Decision}

The last fusion step is the decision. For example, the choice of the class to which a point belongs. This binary decision can be weighted with a quality measurement, allowing its acceptance or its rejection. The most used rule for the probabilistic and Bayesian decision is the maximum a posteriori:

$$
x \in C_{i} \text { si } p\left(x \in C_{i} / I_{1}, \ldots, I_{l}\right)=\max \left\{p\left(x \in C_{k} / I_{1}, \ldots, I_{l}\right), 1 \leq k \leq N\right\}
$$

Several other criteria were developed to adapt the user needs and the decision context as well as possible. Especially, we cite: the maximum probability, the maximum entropy, the maximum hope, the minimal risk, etc.

The next section presents the results corresponding to each processing step and the final fusion results.

\section{Results}

\subsection{Pretreatments results}

\subsubsection{Data description}

The proposed Bayesian fusion approach was applied using seven satellite images covering Tunis City area, North Africa: three ERS images acquired at three different dates (acquisitions relatively close, cf. Fig. 3.) and one Spot4 image containing four spectral bands (cf. Fig. 4.).
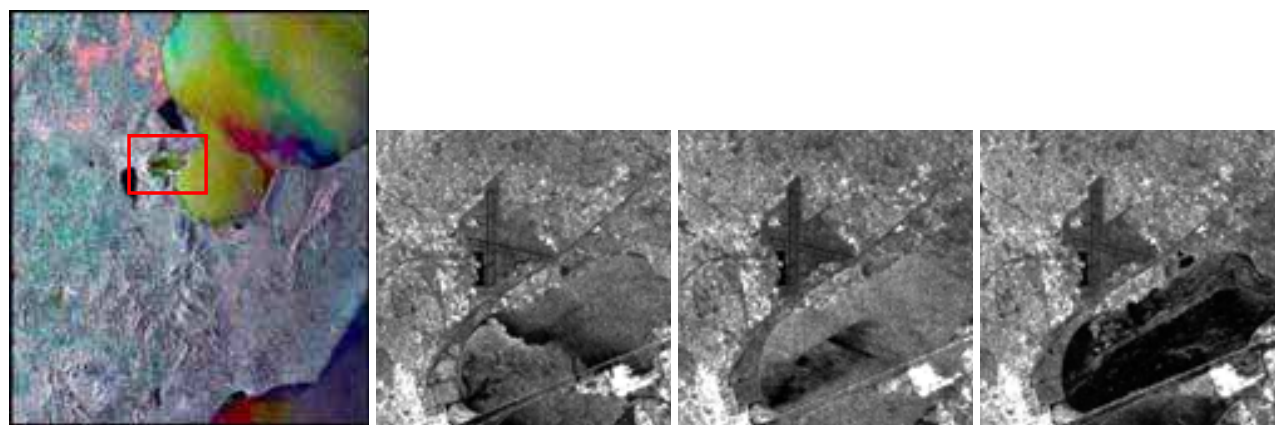

Fig. 3. The multi-temporal radar ERS image composed of three images acquired at three different dates. 

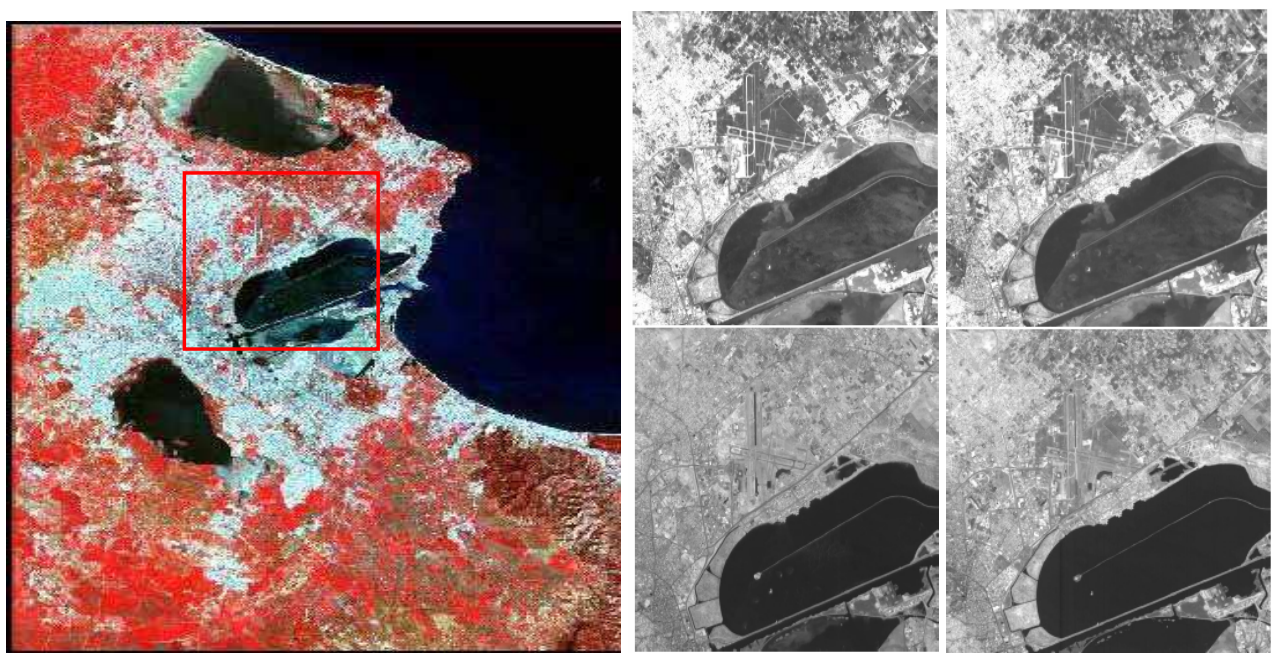

Fig. 4. The Spot4 image composed of four spectral bands.

\subsubsection{Geometric correction}

There are two types of geometric corrections:

- The correction of distortions due to the geometry variations between the ground and the sensor,

- The transformation of the data into true coordinates i.e. into ground geometry coordinates.

We firstly identify several clearly distinct points on the image to be corrected i.e. the radar image. The Spot 4 image is geo-referenced (ground known reference). Then, these points are connected to another set of points selected on the optical image.

Fig. 5. illustrates the geometric correction result applied to the seven images. This preprocessing step is very delicate since its accuracy disturbs fusion results. Registration errors are chosen less than $10^{-2}$.
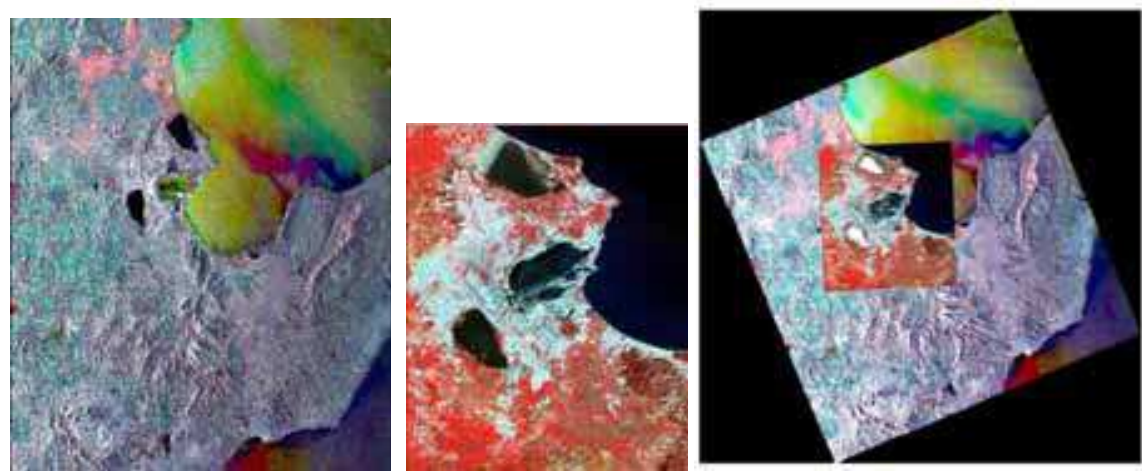

Fig. 5. Result of geometric correction applied between optical and radar images. 


\subsubsection{Speckle filtering}

The proposed fusion approach does not require radar images filtering phase since the radar texture model takes into account the speckle. However, we need filtering for single image classification since it necessitates a strong homogeneity degree inside the classes to be able to distinguish between them.

As explained in paragraph 2.2, the gamma Map filter was retained because it makes it possible to smooth the scene and reduce the speckle noise while preserving the radiometric and textural radar features.
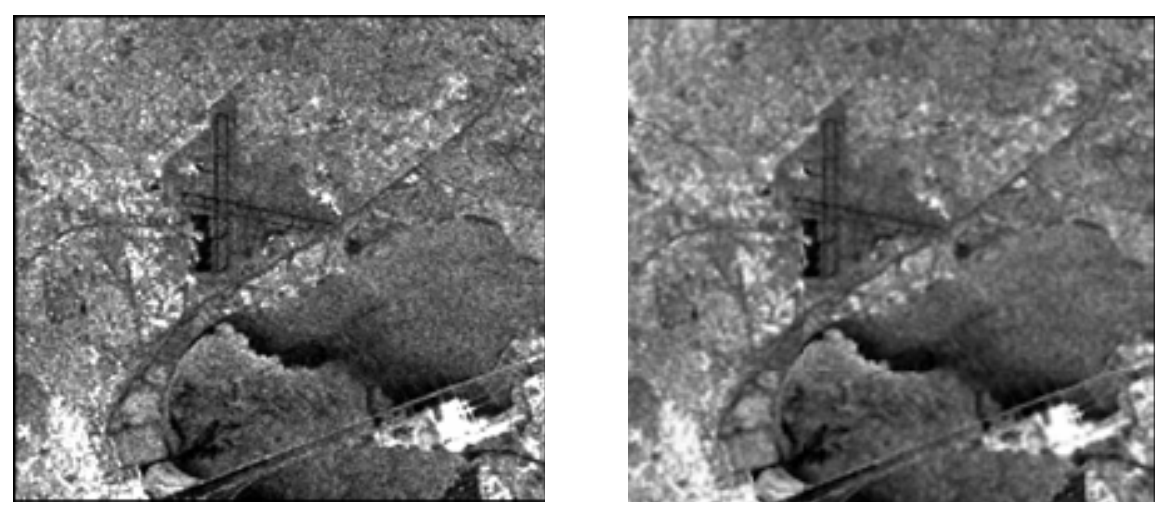

Fig. 6. Filtering results: original radar image (left). Radar image obtained after gamma MAP filtering (right).

The window size of the gamma MAP filter is fixed at 5x5. Fig. 6. shows the radar texture before and after speckle filtering. Radar filtering improves classification results.

\subsubsection{Single image classification results}

FCM classification algorithm was applied on both radar and optical images. To choose the classes number, we study auxiliary data such as maps and High Resolution (HR) images. We identify six classes. For the considered region, there are two types of vegetation: small trees and vegetation under water that we call humid area. There are also two types of urban areas: dense and disperse agglomeration regions.

Fig. 7. and 8. show Fuzzy classification results for the Spot4 four spectral bands and the three ERS radar images. As we can notice the single classification results vary from one image to another. This is due to differences between spectral features and speckle noise. Single classification results give good reason to combine all this kind of information in order to improve land cover classification.

\subsubsection{Parameter estimation results}

EM algorithm (cf. paragraph 2.4.3) was applied for Markovian parameters estimation. Both auto-normal and auto-gamma models parameters exposed in 2.4 are estimated for each classified area. 


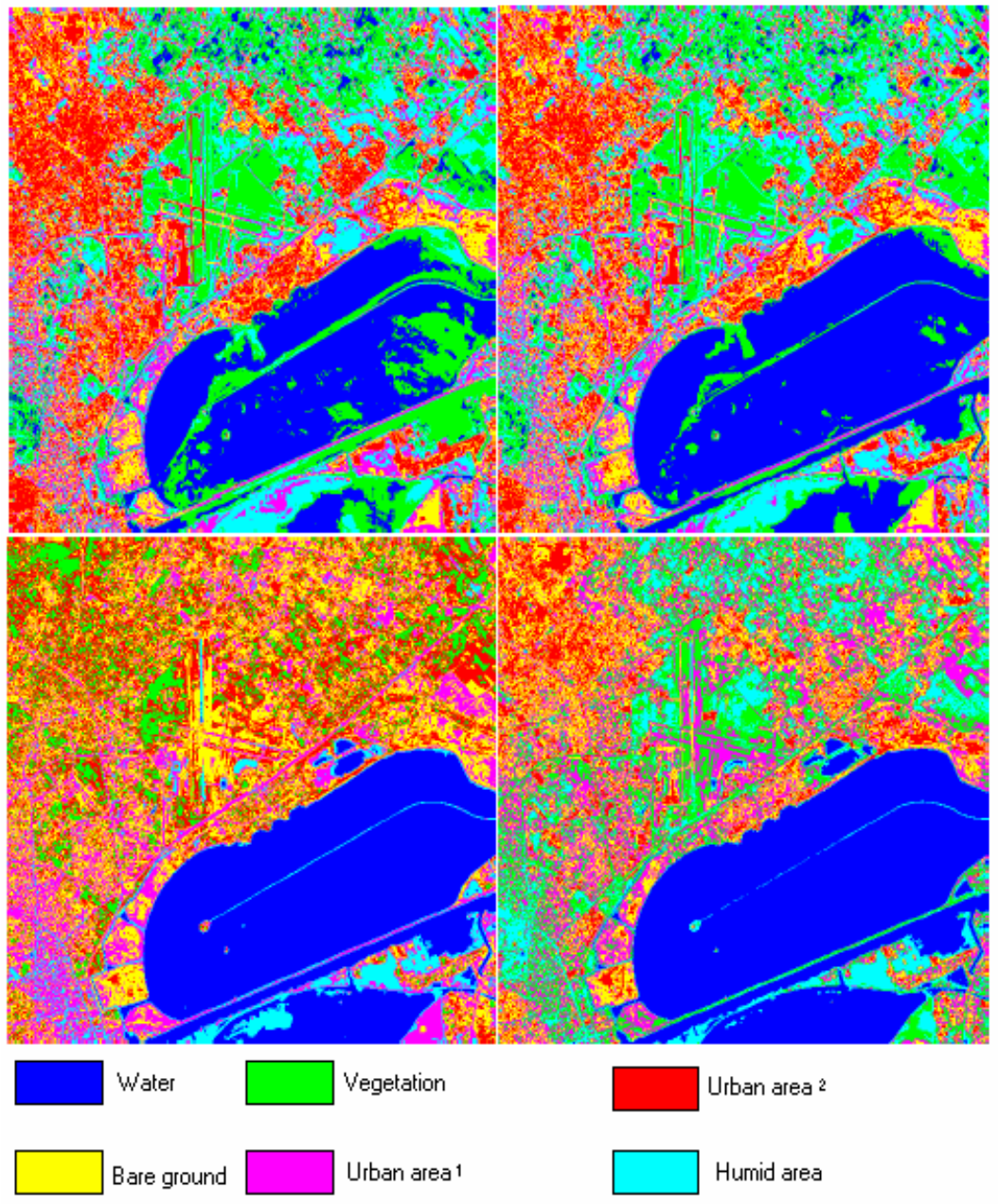

Fig. 7. FCM classification results for Spot4 XS1, XS2, XS3 and XS4 bands (from left to right). 


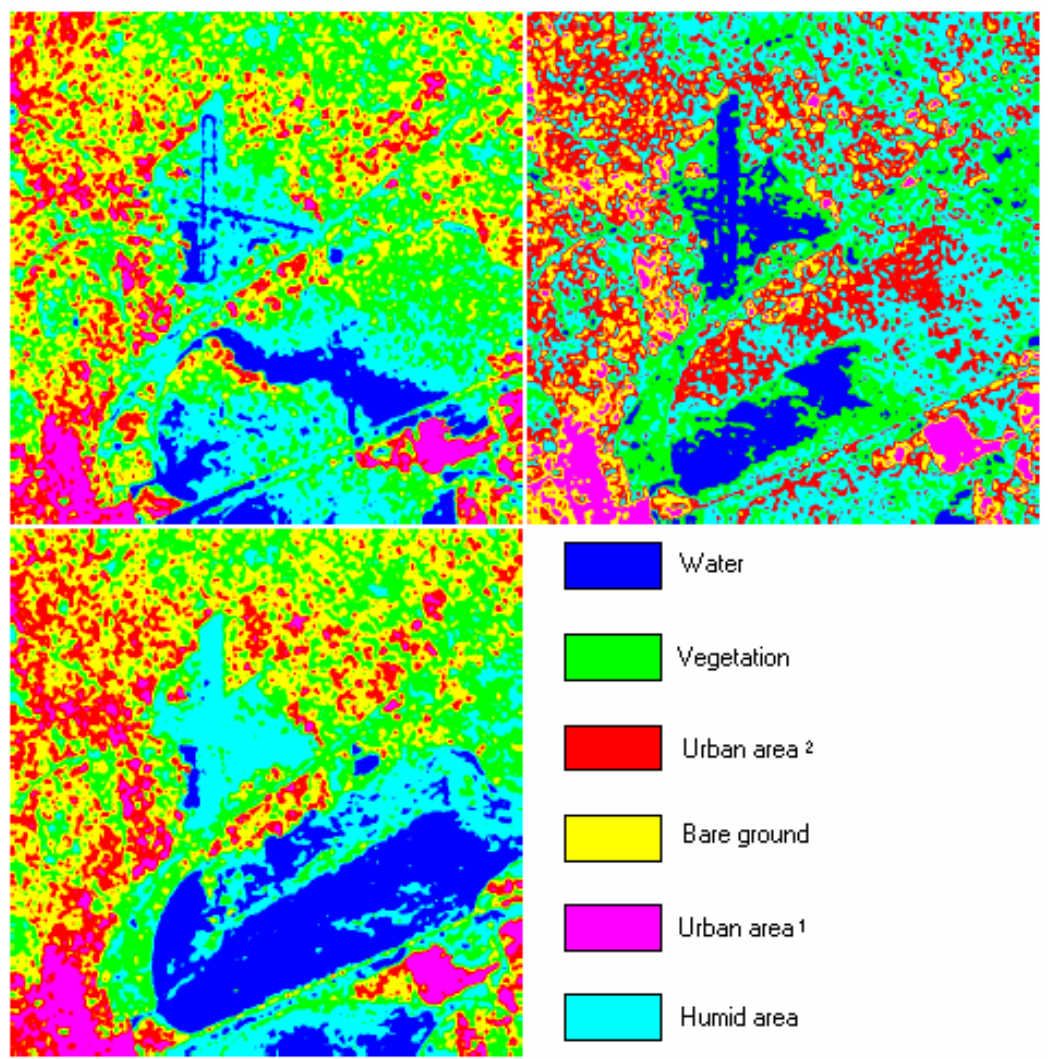

Fig. 8. FCM classification results for ERS images.

\subsection{Fusion results}

In order to highlight the contribution of spatial and contextual information introduced at the modelling level, we will present and compare fusion results obtained with and without spatial information exploitation. The four principal fusion steps are then investigated one by one in both cases.

\subsubsection{Fusion without spatial information}

First, we combine the optical and radar images without taking into account the pixel neighbourhood using Bayesian fusion. The expression of the posterior probability is given by the equation (12). In the case of radar and optical images, it becomes: 


$$
\begin{aligned}
& p\left(x \in C_{i} / I_{\text {radar }_{j=1, \ldots N r}}, I_{\text {optiacl }_{j=1, \ldots N o}}\right) \\
& =\frac{\prod_{j=1}^{N r} p\left(I_{\text {radar }_{j}} / x \in C_{i}\right) \prod_{j=1}^{N o} p\left(I_{\text {optical }_{j}} / x \in C_{i}\right) p\left(x \in C_{i}\right)}{p\left(I_{\text {radar }_{j=1, \ldots N r}}, \ldots, I_{\text {optical }_{j=1 \ldots, N o}}\right)}
\end{aligned}
$$

The modelling step consists in representing the conditional probability related to optical image by a Gaussian distribution and the one related to the radar image by a gamma distribution. The two probabilities are thus written:

$$
p\left(I_{\text {radar }_{j}} / x \in C_{i}\right)=N\left(\mu_{i}, \sigma_{i}^{2}\right)
$$

Where $\mu_{i}$ and $\sigma_{i}^{2}$ represent the Gaussian distribution parameters for the optical image $I_{j}$ and the class $C_{i}$, they correspond respectively to the average and the variance.

$$
p\left(I_{\text {optical }} / x \in C_{i}\right)=\gamma\left(a_{i}, \alpha_{i}\right)
$$

Where, $a_{i}$ and $\alpha_{i}$ represent the gamma distribution parameters for the radar image radar $I_{j}$ and the class $C_{i}$.

Let us notice here, that we assume the sources independence which is justified by different nature of sensors.

Concerning the choice of the prior probability $p\left(x \in C_{i}\right)$, we fixed the same probability for each class. Indeed, since we do not have prior information about the real percentage of each class in the studied zones, one of the prior probabilities can be considered as equally probable.

Other choices can be carried out for the prior probability such as the occupation percentage of each class according to the most reliable image source, the Markovian modelling, etc.

The second step which is the estimation consists in determining for each class $C_{i}, \mu_{I}, \sigma_{i}^{2}, a_{i}$ and $\alpha_{i}$ by likelihood maximization. The combination is done using the Bayesian rule and the decision criterion is the posterior maximum.

\subsubsection{Qualitative evaluation}

We can notice here that the multi-source image fusion allows the characterization of humid and small vegetation dispersed areas inside Tunis City Lake. The fusion of two set of images of different nature highlights the presence of these zones. As we can see from high resolution Google earth image (cf. Fig. 9.), these areas have already existed and are not selected by a single image classification which underline the need of multi-source image classification.

The fusion of the seven images also characterizes better the urban zones and the road network. Indeed, we can observe the good detection of linear and fine structures at the level of the airport crossing raising thus confusion with vegetation areas. Moreover, the bare ground class is not too present after fusion; there is a certain confusion with urban classes, especially around Tunis City Lake.

\subsubsection{Quantitative evaluation}

Beside qualitative results, a manual classified image delimited by the help of higher resolution images, is used to evaluate quantitatively results accuracies. Thus we calculate 


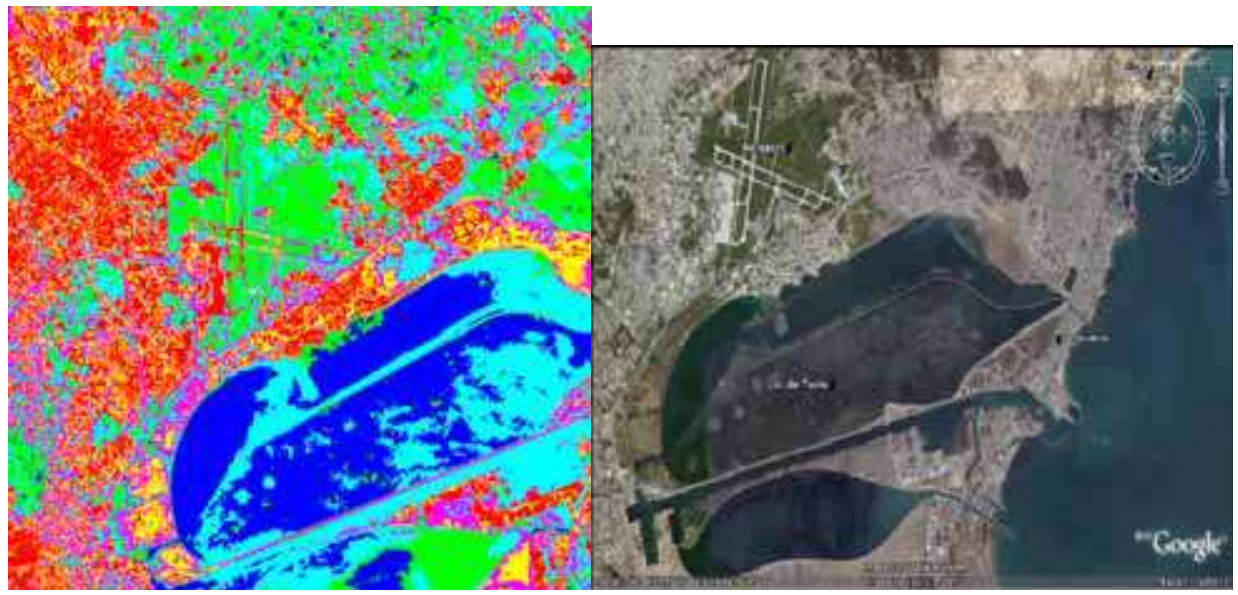

Fig. 9. The fist image corresponds to Bayesian fusion results without spatial information and the second image represents a high resolution Google Earth image.

the confusion matrix [Bloch, 2008] according to the manual classified image (table1). This quantitative measure expresses the good detection and false alarms rates according to each class. As we can see, without taking into acount spatial information, we obtain suffisent results.

\begin{tabular}{|c|c|c|c|c|c|c|}
\hline Water & Vegetation & Urban 1 & $\mathrm{Ba}$ & e ground & Urban 2 & Humid zone \\
\hline Water & $97.50 \%$ & $0.00 \%$ & $1.70 \%$ & $0.00 \%$ & $0.00 \%$ & $2.61 \%$ \\
\hline Vegetation & $0.00 \%$ & $91,65 \%$ & $1.00 \%$ & $1.50 \%$ & $3.10 \%$ & $0.35 \%$ \\
\hline Urban 1 & $0.00 \%$ & $1.50 \%$ & $89 \%$ & $3.40 \%$ & $2.67 \%$ & $3.36 \%$ \\
\hline Bare ground & $0.00 \%$ & $2.55 \%$ & $4.98 \%$ & $91.55 \%$ & 0.10 & 0.15 \\
\hline Urban 2 & $0.00 \%$ & $3.00 \%$ & $1.32 \%$ & $3.20 \%$ & 92.44 & $1.13 \%$ \\
\hline Humid zone & $2.50 \%$ & $1.30 \%$ & $2 \%$ & $0.35 \%$ & $0.79 \%$ & $92.40 \%$ \\
\hline
\end{tabular}

Table 1. The resulted Bayesian fusion confusion matrix. Case of the non exploitation of spatial information.

For the second step, we introduce space information into the fusion process.

\subsubsection{Fusion with spatial information}

The introduction of spatial information is done using the Markovian modelling of each class conditional probability. Besag auto-models are attributed to each source of information. We used auto-normal model for optical images because of optical texture homogeneity and auto-gamma model for non filtered radar images (cf. paragraph 2.4). Indeed, it has been shown that radar speckle texture follows a gamma distribution which has different features compared to a Gaussian distribution. Comparisons between Gaussian and gamma modeling are carried out to highlight the efficiency of gamma modeling in case of radar texture. Thus for optical texture the conditional probability is defined as: 


$$
P\left(I_{\text {optical }_{j}} / x_{s} \in C_{i}\right) \propto \frac{1}{\sqrt{2 \pi \sigma_{i}}} \exp \left(\left(-\frac{1}{2 \sigma_{i}^{2}}\left(x_{s}-\mu_{i}-\sum_{r \in V_{s}} \beta_{i}\left(x_{r}-\mu_{i}\right)\right)\right)^{2}\right)
$$

Where $\mu_{i}, \sigma_{i}$ and $\beta_{i}$ are the Markov Gaussian model parameters for each class and $V_{s}$ is the neighborhood of each site $s$ in the image. As for non filtered radar texture the conditional probability is defined by the following equation:

$$
P\left(I_{\text {radar }_{j}} / x_{s} \in C_{i}\right) \propto \gamma\left(a_{i},\left(\alpha_{i}+\sum_{r \in V_{s}} \beta_{i} x_{r}\right)\right) \propto x_{s}^{a_{i}-1} \exp \left(-x_{s}\left(\alpha_{i}+\sum_{r \in V_{s}} \beta_{i} x_{r}\right)\right)
$$

Where $a_{i}, \alpha_{i}$ and $\beta_{i}$ are the gamma auto-model parameters for each considered class. $V_{s}$ is the neighborhood of each site $s$. We remind here that radar images are not filtered for fusion process as for classification, because the gamma model takes into accounts the speckle granular texture [Belhadj et al., 2000].

The prior probability is chosen as a uniform probability to avoid FCM initial classifications influence in fusion process. The second step of the proposed fusion process consists in Markovian auto-models parameters estimation. Therefore, the parameters $\left(\hat{\mu}_{i}, \hat{\sigma}_{i}, \hat{\beta}_{i}\right)$ for Gaussian model and $\left(\hat{a}_{i}, \hat{\alpha}_{i}, \hat{\beta}_{i}\right)$ for gamma model are estimated using an EM algorithm.

The neighborhood order is fixed at 3 [Meddeb et al., 2007] for both radar and optical images. The fusion combination step is done by multiplying the modeled posterior probability of each source of information following the Bayesian fusion theory. We refer to equation (14) to replace the conditional probability term by its corresponding expression, equation (17) for optical data and equation (18) for non filtered radar texture. Class decision is the last step of the fusion process. It is assured for each pixel using the Maximum A Posteriori probability (MAP) method.

\subsubsection{Qualitative evaluation}

Comparing to single FCM classification and fusion by introducing spatial information results, we point out a clear improvement of class distribution. Indeed, on the one hand, urban zones are better delimited (cf. fig. 10.). On the other hand, humid and vegetation

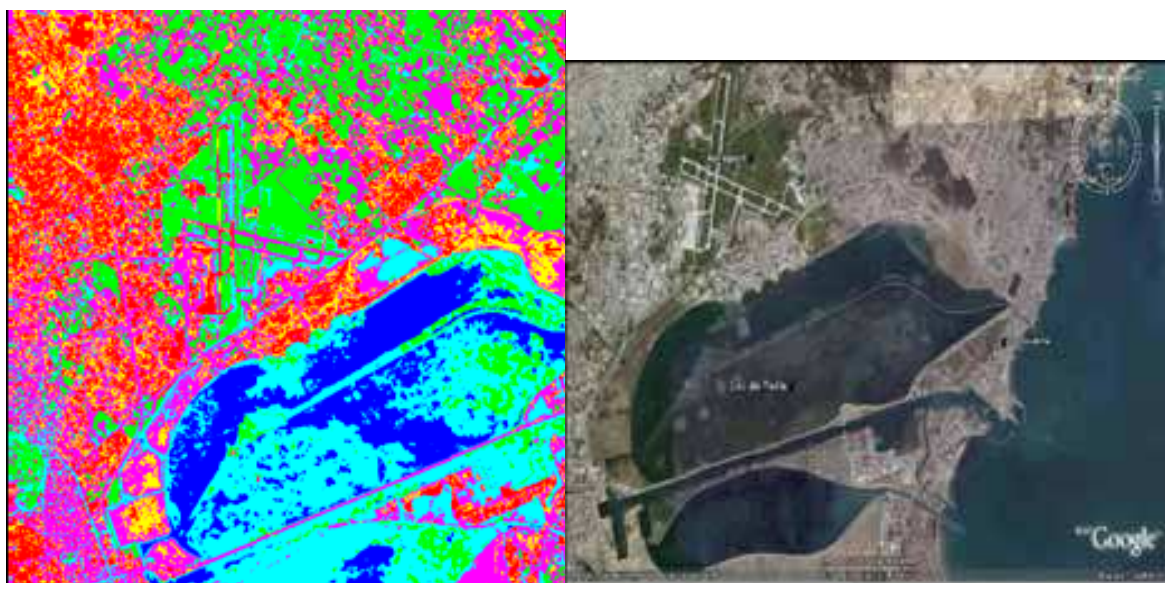

Fig. 10. The fist image corresponds to Bayesian fusion results by introducing the spatial information and the second image represents a high resolution Google Earth image. 
areas in the middle of Tunis City Lake are refined. However, there are some false alarms especially for vegetation areas and missed detections for urban areas.

The urban zones 1 and 2 are quite present on the image with some confusion with the bare ground class which is less present after fusion. Also, the false alarm water areas selected away from Tunis City Lake are not present any more, and confusion with the vegetation was raised.

\subsubsection{Quantitative evaluation}

Looking at the obtained confusion matrix, we notice that diagonal values corresponding to good classification rates are sufficiently important. Besides, false alarm water areas outside Tunis City Lake are removed (comparing to single classification) reducing confusion with vegetation areas. However, there are still confusions between humid and vegetation areas, urban and bare ground areas.

By comparing tables 1 and 2, we notice an improvement for the good classification rate. On the other hand, it is noted that certain false alarms are less important. The introduction of spatial information is then quantitatively justified.

\begin{tabular}{|c|c|c|c|c|c|c|}
\hline Water & Vegetation & Urban 1 & & ground & Urban 2 & Humid zone \\
\hline Water & $98.10 \%$ & $0.00 \%$ & $1.71 \%$ & $0.00 \%$ & $0.00 \%$ & $1.23 \%$ \\
\hline Vegetation & $0.00 \%$ & $92,00 \%$ & $1.00 \%$ & $1.50 \%$ & $2.10 \%$ & $0.00 \%$ \\
\hline Urban 1 & $0.00 \%$ & $1.50 \%$ & $93.88 \%$ & $1.78 \%$ & $0.55 \%$ & $1.20 \%$ \\
\hline Bare ground & $0.00 \%$ & $2.55 \%$ & $2.33 \%$ & $94.22 \%$ & 0.10 & 1.35 \\
\hline Urban 2 & $0.00 \%$ & $3.00 \%$ & $0.15 \%$ & $2.15 \%$ & 96.40 & $0.12 \%$ \\
\hline Humid zone & $1.90 \%$ & $1.30 \%$ & $1.54 \%$ & $0.35 \%$ & $0.85 \%$ & $96.10 \%$ \\
\hline
\end{tabular}

Table 2. The resulted Bayesian fusion confusion matrix.

\section{Conclusion}

Two Besag Markovian auto-models are used to characterize remote sensing data issued from two different sensors. Gaussian model is applied on optical images whereas gamma model is used to represent radar images. For both models, an optimal Markov neighborhood order is used. Confusion matrix rates show that the proposed Bayesian fusion approach gives sufficient results according to single FCM classification. For future works and in order to improve the obtained results, we can introduce a reliability degree to each source of information in a fuzzy Bayesian fusion framework.

\section{References}

Baraldi, A.; Panniggiani, F. (1995). A refined gamma MAP SAR speckle filter with improved geometrical adaptivity, IEEE Transaction on Geoscience and Remote Sensing, pp. 1245 - 1257, Sep 1995.

Bazi, Y. \& Melgani, F. (2006). Toward an Optimal SVM Classification System for Hyperspectral Remote Sensing Images, IEEE Transaction on Geoscience and Remote Sensing, pp. 3374-3385, Vol. 44, Issue 11, Nov. 2006. 
Benediktsson, J., Swain, P. \& Ersoy, O. (1990). Neural network approaches versus statistical methods in classification of multisource remote sensing data, IEEE Transaction on Geoscience and Remote Sensing, Vol. 28, Nº4, pp. 540-552, july 1990.

Besag, J. (1974). Spatial interaction and the statistical analysis of lattice systems, Journal of the Royal Statistical Society, Series B, Vol. 36, pp.192-236.

Bloch, A. (2008). Information fusion in signal and image processing major probabilistic and non-probabilistic numerical approaches, ISTE John Wiley \& Sons Ed., ISBN: 1-84821019-1, Janvier 2008.

Bezdek, J.C.; R. Ehrlich \& Fall W. (1984). FCM: the fuzzy c-means clustering algorithm, Computers and Geoscience, Vol. 10, pp. 191-203.

Dempster, A. P.; Laird N. M. \& Rubin D. B. (1977). Maximum Likelihood from Incomplete Data via the EM Algorithm, Journal of the Royal Statistical Society, B, Vol.. 39, N 1, pp. 138.

Descombes, X.; Sigelle, M. \& Préteux F.(1999). Estimating Gaussian Markov random field parameters in a nonstationary framework: application to remote sensing imaging. IEEE Trans. on Image Processing, Vol. 8, N 4, pp. 490-503.

Hogg, R.; McKean, J. \& Craig, A. (2005). Introduction to Mathematical Statistics, 5th Edition, Upper Saddle River, NJ: Pearson Prentice Hall, 2005.

Hong, T. D.; Schowengerdt, R. A. (2005). A Robust Technique for Precise Registration of Radar and Optical Satellite Images, Photogrammetric Engineering \& Remote Sensing, Vol. 71, No. 5, pp. 585-593, May 2005.

Hosomura, T. \& Jayasekera, C.W. (1993). Speckle filtering and texture analysis in SAR images, Geoscience and Remote Sensing Symposium, Better Understanding of Earth Environment., Vol. 3, pp. 1423 - 1425, Aug 1993.

Klein, L. A. (2004). Sensor and Data Fusion: A Tool for Information Assessment and Decision Making, SPIE Press, 342pp, ISBN: 0819454354, July 2004.

Lee, J. S.; Jurkevich, L.; Dewaele, P.; Wambacq, P. \& Oosterlinck, A. (1994). Speckle filtering of synthetic aperture radar images: A review, Remote Sensing Reviews, Vol. 8, Issue 4, pp. 313 - 340, January 1994.

Lorette, A.; Descombes, X. \& Zerubia J. (2000). Urban areas extraction based on texture analysis through a Markovian modelling, Int. Journal of Computer Vision, Vol. 36, N³, pp. 219-234.

Maitre, H. (2000). Traitement des images de RSO, Traité IC2, série traitement du signal et de l'image, ISBN: 2-7462-0155-0, Editions hermes-sciences 2000.

Meddeb, A.; Chaabane, F. \& Belhadj, Z. (2007). Réflexion sur le choix de l'ordre de voisinage pour la modélisation par les champs de Markov de la texture SAR, Traitements et Analyse d'Images, Méthodes et Applications, TAIMA'07, Hammamet, Mai 2007.

Milisavljevi'C, N.; Bloch, I.\& Acheroy, M. (2008). Multi-Sensor Data Fusion Based on Belief Functions and Possibility Theory: Close Range Antipersonnel Mine Detection and Remote Sensing Mined Area Reduction, in Humanitarian Demining: Innovative Solutions and the Challenge of Technology, chap. 4, pp. 392-418, M. K. Habib Ed., ARS I-Tech Education and Publishing, Vienna, Austria.

Milisavljevi'C, N. \& Bloch, I. (2009). Possibilistic and fuzzy multi-sensor fusion for humanitarian mine action, in Advances in Geoscience and Remote Sensing, chap. 23, pp. 491-504, Gary Jedlovec Ed., InTech Croatia.

Mitchell, H.B. (2007). Multi-Sensor Data Fusion, springer Verlag, ISBN: 3540714634, jully 2007. 
Phillips, S. (2002). Reducing the computation time of the Isodata and K-means unsupervised classification algorithms, IEEE International Geoscience and Remote Sensing Symposium, IGARSS '02, Vol. 3, pp. 1627 - 1629, Nov. 2002.

Rui-hui, P.; Shu-zong, W.; Xiang-wei, W. \& Yong-sheng, L. (2009). Modelling of correlated gamma-distributed texture based on spherically invariant random process, IEEE International Conference on Intelligent Computing and Intelligent Systems, ICIS, pp. 53 58, Shanghai, Nov. 2009.

Shabou, A. \& Tupin F. \& Chaabane, F. (2007). Similarity measures between SAR and optical data, IEEE International Geoscience and Remote Sensing Symposium (IGARSS'07), Barcelona, Spain, July 2007.

Tang, X.O. (1998). Multiple Competitive Learning Network Fusion for Object Classification, SMCB, Vol. 28, N 4, pp. 532-543, August 1998.

Wang, F. (1990). Fuzzy Supervised Classification of remote Sensing Images, IEEE Transaction on Geoscience and Remote Sensing. Vol. 28, No. 2, Mars 1990.

Zitova, B.; Flusser, J. (2003). Image Registration Methods: A Survey, Image and Vision Computing, Vol. 21, pp. 977-1000, Elsevier, June 2003. 


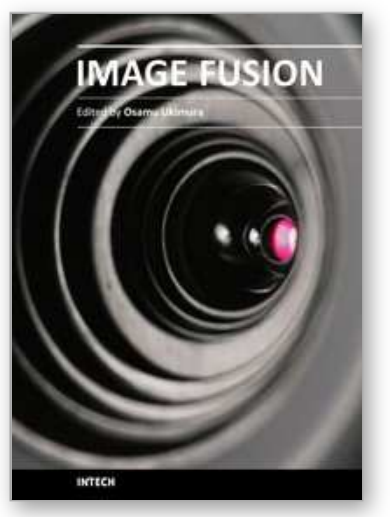

\author{
Image Fusion \\ Edited by Osamu Ukimura
}

ISBN 978-953-307-679-9

Hard cover, 428 pages

Publisher InTech

Published online 12, January, 2011

Published in print edition January, 2011

Image fusion technology has successfully contributed to various fields such as medical diagnosis and navigation, surveillance systems, remote sensing, digital cameras, military applications, computer vision, etc. Image fusion aims to generate a fused single image which contains more precise reliable visualization of the objects than any source image of them. This book presents various recent advances in research and development in the field of image fusion. It has been created through the diligence and creativity of some of the most accomplished experts in various fields.

\title{
How to reference
}

In order to correctly reference this scholarly work, feel free to copy and paste the following:

Chaabane Ferdaous (2011). Remote Sensing Image Fusion for Unsupervised Land Cover Classification, Image Fusion, Osamu Ukimura (Ed.), ISBN: 978-953-307-679-9, InTech, Available from:

http://www.intechopen.com/books/image-fusion/remote-sensing-image-fusion-for-unsupervised-land-coverclassification

\section{INTECH}

open science / open minds

\section{InTech Europe}

University Campus STeP Ri

Slavka Krautzeka 83/A

51000 Rijeka, Croatia

Phone: +385 (51) 770447

Fax: +385 (51) 686166

www.intechopen.com

\section{InTech China}

Unit 405, Office Block, Hotel Equatorial Shanghai

No.65, Yan An Road (West), Shanghai, 200040, China

中国上海市延安西路65号上海国际贵都大饭店办公楼405单元

Phone: +86-21-62489820

Fax: +86-21-62489821 
(C) 2011 The Author(s). Licensee IntechOpen. This chapter is distributed under the terms of the Creative Commons Attribution-NonCommercialShareAlike-3.0 License, which permits use, distribution and reproduction for non-commercial purposes, provided the original is properly cited and derivative works building on this content are distributed under the same license. 